\title{
Malignant melanoma arising in congenital melanocytic nevi: clinical and dermoscopic challenges
}

\author{
Fatma Pelin Cengiz, Nazan Emiroglu, Anil Gulsel Bahali, Ozlem Su, Nahide Onsun
}

Department of Dermatology, Bezmialem Vakif University, Istanbul, Turkey

Corresponding author: Dr. Fatma Pelin Cengiz, E-mail: fpelinozgen@hotmail.com

\begin{abstract}
Congenital melanocytic nevi $(\mathrm{CMN})$ are visible pigmented lesions in the skin that are present at birth. CMN are benign malformations resulting from defective development of melanocyte precursors in the embryo. Six MMs from six patients were analyzed by clinical and dermoscopic examination. Of the patients, $33.3 \%$ were female $(\mathrm{N}=2)$ and $66.6 \%$ were male $(\mathrm{N}=4)$. Of the MMs, four $(66.6 \%)$ were superficial spreading $\mathrm{MM}$ and two $(33.3 \%)$ were in situ MM. A reticular pattern was present in the MMs of three patients (50\%), a homogeneous pattern was present in the other patients (50\%) at the base of the MMs. Superficial spreading melanomas and in situ melanomas with atypical dots and globules and a blue-white veil were the most common dermoscopic features of MMs found in CMN.
\end{abstract}

Key words: Melanoma; Dermoscopy; Nevi

\section{INTRODUCTION}

Congenital nevi result from a proliferation of benign melanocytes in the dermis or epidermis or both. Congenital nevi may develop during the first two years of life if they are not apparent at birth [1]. These lesions are described as 'congenital nevus-like nevi' or 'tardive congenital nevi' by some authors [2]. Congenital nevi are present in 1:20000 of newborn infants [3].

There is a low risk of developing malignant melanoma (MM) in small to medium CMN. Up to $1 \%$ of patients with small or medium CMNs will develop MM over a lifetime [3]. Our aim was to investigate the dermoscopic patterns of MM that developed in congenital melanocytic nevi $(\mathrm{CMN})$ to identify specific dermoscopic features.

\section{CASE REPORTS}

Six patients at the Department of Dermatology at Bezmialem Vakif University Hospital in Istanbul over five years were recruited for the study. Essential inclusion criteria were the presence of MM and the ability of the participants to state with certainty whether MM arose on a small or medium nevus that was present at birth or that appeared during the first two years of life. Dermoscopic images of all lesions were acquired at a $30 \mathrm{X}$ magnification and were stored in a digital imaging system (Fotofinder, Digital Dermoscopy).

Dermoscopic patterns were classified as reticular, globular, cobblestone, or homogeneous. A small congenital nevus was defined as having a diameter of less than $1.5 \mathrm{~cm}$, and a medium congenital nevus was defined as having a diameter of more than $1.5 \mathrm{~cm}$ but less than $20 \mathrm{~cm}$.

A descriptive analysis of the sample set was performed, which included calculation of percentages for categorical variables.

Clinical and dermoscopic examinations were performed on six patients.

\section{Descriptive Results}

There were four male patients $(66.6 \%)$ and two female patients $(33.3 \%)$ whose ages ranged from 40 to 82 years.

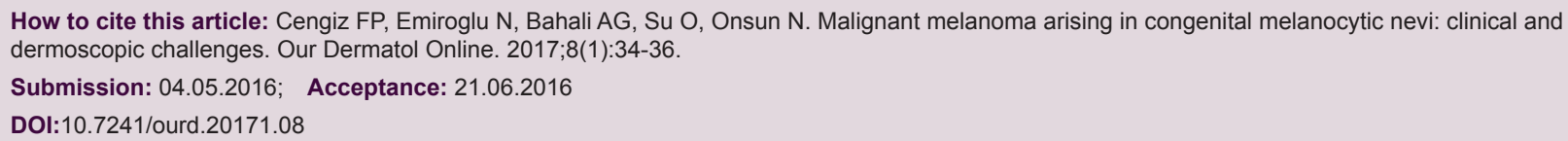


The mean age of the patients was $55 \pm 7$ years. One patient had MM on the shoulder, one patient had MM on a limb, three patients had MM on the back, and one patient had MM on the forearm. Of the MMs, four developed on medium nevi and two developed on small nevi.

Changes in nevus were reported over the past six months (two patients), one year (one patient), two years (two patients), and five years (one patient).

\section{Dermoscopic Patterns}

On dermoscopic examination, we found the presence of a reticular pattern $(50 \% ; \mathrm{N}=3)$ and a homogeneous pattern (50\%; $\mathrm{N}=3$ ) (Fig. 1). Perifollicular hypopigmentation, milia-like cysts, an atypical pigment network and radial streaks were present in one MM (16.6\%) (Fig. 2). Hypertrichosis and focal hypopigmentation were not observed in any lesion. Atypical dots and globules, blue-white veil were observed in three MMs (50\%) (Fig. 3). Regression and atypical vascular structures were present in one MM $(16.6 \%)$.

\section{DISCUSSION}

The congenital melanocytic nevus presents as a circumscribed, light brown to black patch or plaque, heterogeneous in consistency, covering any size and any part of the body. Most instances of small CMN usually appear during childhood or puberty [4]. Large CMN have a higher risk of developing MM than small and medium nevi. MM arising in CMN usually develop at younger ages and are located superficially within small or medium CMN and deep within large CMN. Two important meta-analyses were conducted to determine the relationship between the size of $\mathrm{CMN}$ and future development of MM [5,6]. In one of these studies, ten of the 432 patients developed MM within their giant congenital nevi. Krengel et al. analyzed 14 studies with a combined 6,571 CMN patients who were followed for 3.4-23.7 years and found that $46(0.7 \%)$ patients developed a total of 49 MMs (mean age at diagnosis: 15.5 years; median age: 7 years). The authors found an increased relative risk of developing MM during childhood and adolescence [6].

Many of the nevi termed 'dysplastic' or 'atypical' are actually small CMN [4]. Multiple CMN may reflect a mosaic RASopathy as a result of postzygotic activating mutations in NRAS [7], and patients with small

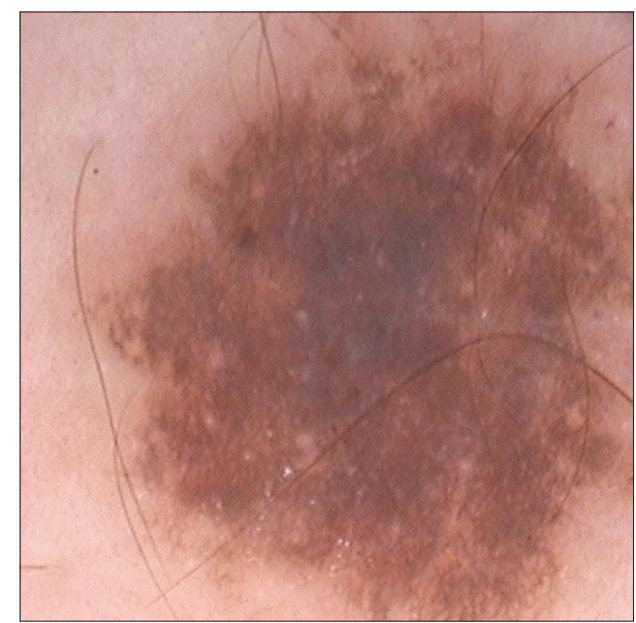

Figure 1: Atypical network, milia-like cysts, perifollicular hypopigmentation.

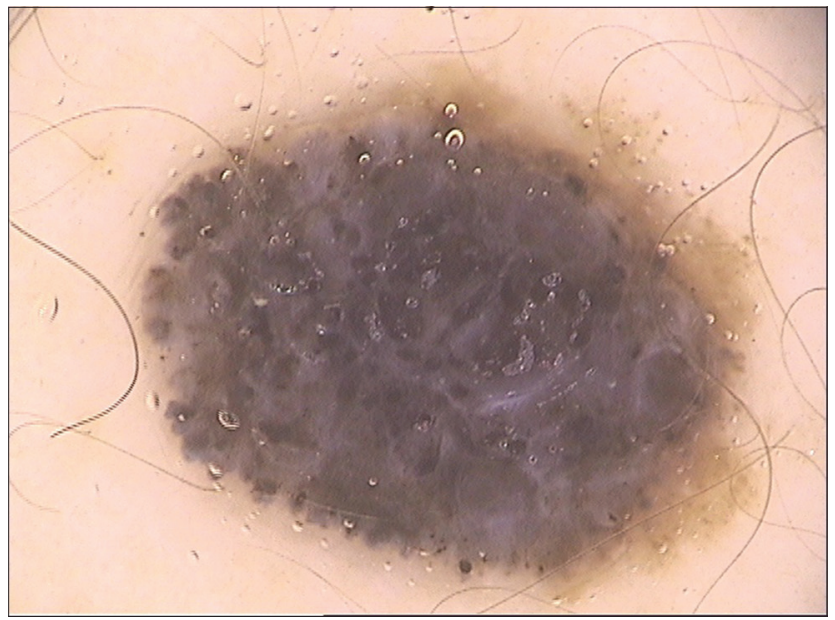

Figure 2: Spitzoid pattern, black structureless area.

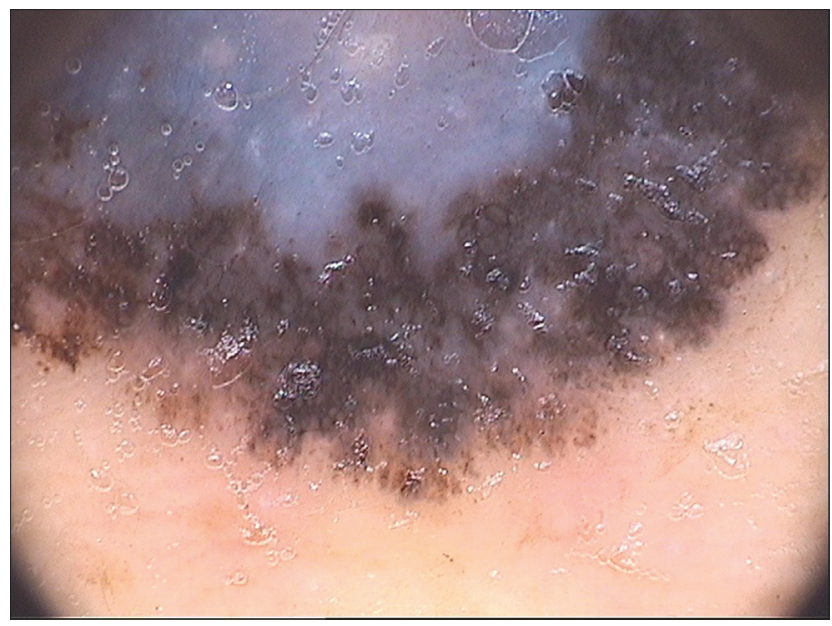

Figure 3: Radial streaks, blue-white veil, atypical dots.

CMN have a higher risk of MM. The lifetime risk of developing MM is estimated to be 1 in 100 for patients with small and medium CMN [8]. Annual clinical and digital dermoscopic examination is indicated for these 
patients. Therefore, the physician should know the dermoscopic features of CMN to avoid unnecessary excisions, and to recognize MM when it begins to develop.

One patient, who attended follow-up visits for annual dermoscopic examinations at our clinic, was concerned about pigmentary changes in his nevus an he was diagnosed with in situ MM. The other patients also attended our outpatient clinic for examination of the changes in their nevi. The patients whose nevi had Breslow thickness of $6.12 \mathrm{~mm}$ and $13.73 \mathrm{~mm}$ died within the first year after diagnosis.

Although rare, the occurrence of MM in small congenital nevi has been described in adults and children $[9,10]$. To the best of our knowledge, the work reported herein is the first case series describing patients with MM that developed in small or medium congenital nevi.

Atypical dots and globules and a blue-white veil were the most common dermoscopic features of CMN found in our study:

- Superficial spreading MM and in situ MM were the only types of MM found in the six CMN patients examined;

- The dermoscopic features of CMN that developed MM were reticular and homogeneous patterns;

- The dermoscopic features of MM that developed in CMN were atypical dots and globules, and a bluewhite veil; and

- The results may help to define accurately the dermoscopic features of congenital melanocytic nevi.

\section{ACKNOWLEDGMENTS}

We thank our patients, who were essential to this work, for their participation.

\section{REFERENCES}

1. Marghoob AA. Congenital melanocytic nevi. Evaluation and management. Dermatol Clin. 2002;20:607-16.

2. Makkar HS, Frieden IJ. Congenital melanocytic nevi: an update for the pediatrician. Curr Opin Pediatr. 2002;14:397-403.

3. Alikhan A, Ibrahimi OA, Eisen DB. Congenital melanocytic nevi: where are we now? Part I. Clinical presentation, epidemiology, pathogenesis, histology, malignant transformation, and neurocutaneous melanosis. J Am Acad Dermatol. 2012;67:495. e1-17 [quiz: 512-4].

4. Kittler H, Rosendahl C, Cameron A, Tschandl P. Dermatoscopy. An algorithmic method based on pattern analysis. 2011 Edition.P:29-35.

5. Watt AJ, Kotsis SV, Chung KC. Risk of melanoma arising in large congenital melanocytic nevi: a systematic review. Plast Reconstr Surg. 2004;113:1968-74.

6. Krengel S, Hauschild A, Schäfer T. Melanoma risk in congenital melanocytic nevi: a systematic review. Br J Dermatol. 2006;155:1-8.

7. Waelchi R, Williams J, Cole T, Dattani M, Hindmarsh P, Kennedy H, et al. Growth and hormonal profiling in children with congenital melanocytic nevi. Br J Dermatol. 2015;173:1366-7.

8. Sahin S, Levin L, Kopf AW, Rao BK, Triola M, Koenig K, et al. Risk of melanoma in medium-sized congenital melanocytic nevi: a follow-up study. J Am Acad Dermatol. 1998;39:428-33.

9. Betti R, Inselvini E, Vergani R, Crosti C. Small congenital nevi associated with melanoma: Case reports and considerations. J Dermatol. 2000;27:583-90.

10. Zangari A, Ilari M, Nino F, Ascanio M. Report of a malignant melanoma arising in a small congenital nevus in a 3-yearold child. J Indian Assoc Pediatr Surg. 2013;18:165-6.

Copyright by Fatma Pelin Cengiz, et al. This is an open access article distributed under the terms of the Creative Commons Attribution License, which permits unrestricted use, distribution, and reproduction in any medium, provided the original author and source are credited.

Source of Support: Nil, Conflict of Interest: None declared. 